\title{
On Solvability of an Inverse Boundary Value Problem for Pseudo Hyperbolic Equation of the Fourth Order
}

\author{
Yashar T. Mehraliyev ${ }^{1} \&$ Afaq F. Huseynova ${ }^{2}$ \\ ${ }^{1}$ Department of differential and inteqral equations, Baku State University, Baku, Azerbaijan \\ Correspondence: Yashar Mehraliyev, Department of differential and inteqral equations, Baku State University, \\ Baku, AZ1148, Azerbaijan. Tel: 994-12-539-0821. E-mail: yashar_aze@mail.ru
}

Received: February 25, 2015 Accepted: March 14, 2015 Online Published: April 7, 2015

doi:10.5539/jmr.v7n2p101

URL: http://dx.doi.org/10.5539/jmr.v7n2p101

\begin{abstract}
We analyze the solvability of the inverse boundary problem with an unknown coefficient depended on time for the pseudo hyperbolic equation of fourth order with periodic and integral conditions. The initial problem is reduced to an equivalent problem. With the help of the Fourier method, the equivalent problem is reduced to a system of integral equations. The existence and uniqueness of the solution of the integral equations is proved. The obtained solution of the integral equations is also the only solution to the equivalent problem. Basing on the equivalence of the problems, the theorem of the existence and uniqueness of the classical solutions of the original problem is proved.
\end{abstract}

Keywords: inverse boundary problem, pseudo hyperbolic equation, method Fourier, classic solution

\section{Introduction}

There are many cases where the needs of the practice bring about the problems of determining coefficients or the right hand side of differential equations from some knowledge of its solutions. Such problems are called inverse boundary value problems of mathematical physics. Inverse boundary value problems arise in various areas of human activity such as seismology, mineral exploration, biology, medicine, quality control in industry etc., which makes them an active field of contemporary mathematics.

The inverse problems are favorably developing section of up-to-date mathematics. Recently, the inverse problems are widely applied in various fields of science.

Different inverse problems for various types of partial differential equations have been studied in many papers. First of all we note the papers of ( Tikhonov , 1943), ( Lavrentyev, 1964), ( Lavrentyev \& Romanov, 1980) , ( Ivanov, Vasin \& Tanina, 1978), (Denisov, 1994) and their followers.

In searching of local and non-local boundary value problems for pseudohyperbolic equations practical and theoretical interests assume great importance and is more actively studied now days.

In this paper, due to the (Mehraliyev, 2011)-( Mehraliyev, 2012), we proved the existence and uniqueness of the solution of the inverse boundary value problem for the pseudohyperbolic equation of fourth order with periodic and integral conditions.

\section{Problem Statement and Its Reduction to Equivalent Problem}

Lets consider for the equation (Gabov \& Orazov,1986)

$$
u_{t t}(x, t)-u_{t t x x}(x, t)-u_{x x}(x, t)=a(t) u(x, t)+f(x, t)
$$

in the domain $D_{T}=\{(x, t): 0 \leq x \leq 1, \quad 0 \leq t \leq T\}$ an inverse boundary problem with initial conditions

$$
u(x, 0)=\varphi(x), \quad u_{t}(x, 0)=\psi(x) \quad(0 \leq x \leq 1),
$$

the periodic condition

$$
u(0, t)=u(1, t) \quad(0 \leq t \leq T)
$$


the non-local integral condition

$$
\int_{0}^{1} u(x, t) d x=0 \quad(0 \leq t \leq T)
$$

and with additional condition

$$
u\left(x_{0}, t\right)=h(t) \quad(0 \leq t \leq T),
$$

where $x_{0} \in(0,1)$ are the given number, $f(x, t), \varphi(x), \psi(x), h(t)$ are the given functions, and $u(x, t), a(t)$ are the required functions.

The condition (4) is a non-local integral condition of first kind, i.e. the one not involving values of unknown functions at the domains boundary points.

Definition. The classic solution of problem (1) - (5) is the pair $\{u(x, t), a(t)\}$ of the functions $u(x, t)$ and a(t) with the following properties:

1)the function $u(x, t)$ is continuous in $D_{T}$ together with all its derivatives contained in equation (1);

2)the function a(t) is continuous on $[0, T]$;

3) all the conditions of (1) - (5) are satisfied in the ordinary sense.

The following lemma is valid.

Lemma 1 Let $f(x, t) \in C\left(D_{T}\right), \int_{0}^{1} f(x, t) d x=0(0 \leq t \leq T) \quad \varphi(x), \psi(x) \in C^{1}[0,1], \quad h(t) \in C^{2}[0, T], \quad h(t) \neq$ $0(0 \leq t \leq T)$ and

$$
\begin{gathered}
\varphi^{\prime}(0)=\varphi^{\prime}(1), \quad \psi^{\prime}(0)=\psi^{\prime}(1), \\
\int_{0}^{1} \varphi(x) d x=0, \quad \int_{0}^{1} \psi(x) d x=0, \quad \varphi\left(x_{0}\right)=h(0), \quad \psi\left(x_{0}\right)=h^{\prime}(0) .
\end{gathered}
$$

Then the problem on finding the classic solution of problem (1) - (5) is equivalent to the problem on defining of the function $u(x, t)$ and $a(t)$, possessing the properties 1) and 2) of definition of the classic solution of problem (1) - (5), from relations (1) - (3) and satisfying

$$
\begin{gathered}
u_{x}(0, t)=u_{x}(1, t) \quad(0 \leq t \leq T), \\
h^{\prime \prime}(t)-u_{t t x x}\left(x_{0}, t\right)-u_{x x}\left(x_{0}, t\right)=a(t) h(t)+f\left(x_{0}, t\right) \quad(0 \leq t \leq T) .
\end{gathered}
$$

Proof. Let $\{u(x, t), a(t)\}$ be a classical solution to the problem (1) - (5). Integrating equation (1) with respect to $x$ from 0 to 1 , we have

$$
\begin{gathered}
\frac{d^{2}}{d t^{2}} \int_{0}^{1} u(x, t) d x-\frac{d^{2}}{d t^{2}}\left(u_{x}(1, t)-u_{x}(0, t)\right)- \\
-\left(u_{x}(1, t)-u_{x}(0, t)\right)=a(t) \int_{0}^{1} u(x, t) d x+\int_{0}^{1} f(x, t) d x \quad(0 \leq t \leq T) .
\end{gathered}
$$

Taking into account that $\int_{0}^{1} f(x, t) d x=0 \quad(0 \leq t \leq T)$ and (4), we find that

$$
\frac{d^{2}}{d t^{2}}\left(u_{x}(1, t)-u_{x}(0, t)\right)+\left(u_{x}(1, t)-u_{x}(0, t)\right)=0 \quad(0 \leq t \leq T) .
$$

By (2) and $\varphi^{\prime}(0)=\varphi^{\prime}(1), \quad \psi^{\prime}(0)=\psi^{\prime}(1)$ we obtain

$$
\begin{gathered}
u_{x}(1,0)-u_{x}(0,0)=\varphi^{\prime}(1)-\varphi^{\prime}(0)=0, \\
u_{t x}(1,0)-u_{t x}(0,0)=\psi^{\prime}(1)-\psi^{\prime}(0)=0 .
\end{gathered}
$$

Since problem (9), (10)has only a trivial solution, we have $u_{x}(1, t)-u_{x}(0, t)=0$, i.e. the condition (6) is fulfilled. 
Assume now that $h(t) \in C^{2}[0, T]$. Differentiating (5) twice, we get

$$
u_{t}\left(x_{0}, t\right)=h^{\prime}(t), \quad u_{t t}\left(x_{0}, t\right)=h^{\prime \prime}(t) \quad(0 \leq t \leq T)
$$

It follows from (1) that

$$
u_{t t}\left(x_{0}, t\right)-u_{t t x x}\left(x_{0}, t\right)-u_{x x}\left(x_{0}, t\right)=a(t) u\left(x_{0}, t\right)+f\left(x_{0}, t\right) \quad(0 \leq t \leq T) .
$$

Hence, taking into account (5) and (11), we conclude that (7) is fulfilled.

Now suppose that $\{u(x, t), a(t)\}$ is a solution of problem (1) - (3), (6), (7), then from (8) and (6) we find that

$$
\frac{d^{2}}{d t^{2}} \int_{0}^{1} u(x, t) d x-a(t) \int_{0}^{1} u(x, t) d x=0 \quad(0 \leq t \leq T) .
$$

By (2) and $\int_{0}^{1} \varphi(x) d x=0, \quad \int_{0}^{1} \psi(x) d x=0$, it is obvious that

$$
\int_{0}^{1} u(x, 0) d x=\int_{0}^{1} \varphi(x) d x=0, \quad \int_{0}^{1} u_{t}(x, 0) d x=\int_{0}^{1} \psi(x) d x=0 .
$$

Since the problem (13), (14) has only a trivial solution, $\int_{0}^{1} u(x, t) d x=0 \quad(0 \leq t \leq T)$, i.e. the condition (4) is fulfilled.

From (7) and (12) we obtain

$$
\frac{d^{2}}{d t^{2}}\left(u\left(x_{0}, t\right)-h(t)\right)=a(t)\left(u\left(x_{0}, t\right)-h(t)\right) \quad(0 \leq t \leq T) .
$$

By (2) and $\varphi\left(x_{0}\right)=h(0), \psi\left(x_{0}\right)=h^{\prime}(0)$ we have

$$
\left\{\begin{array}{l}
u\left(x_{0}, 0\right)-h(0)=\varphi\left(x_{0}\right)-h(0)=0, \\
u_{t}\left(x_{0}, 0\right)-h^{\prime}(0)=\psi\left(x_{0}\right)-h^{\prime}(0)=0 .
\end{array}\right.
$$

From (15) and (16) we conclude that the condition (5) is fulfilled. The lemma is proved.

\section{Investigation of the Existence and Uniqueness of the Classic Solution of the Inverse Boundary Value Problem}

It is known (Budak, Samarskii \& Tikhonov, 1972) that the system

$$
1, \cos \lambda_{1} x, \sin \lambda_{1} x, \ldots, \cos \lambda_{k} x, \sin \lambda_{k} x, \ldots
$$

is a basis in $L_{2}(0,1)$, where $\lambda_{k}=2 k \pi \quad(k=1,2, \ldots)$. Therefore, it is obvious that for each solution $\{u(x, t), a(t)\}$ to the problem (1) - (3), (6), (7) its first component $u(x, t)$ has the form:

$$
u(x, t)=\sum_{k=0}^{\infty} u_{1 k}(t) \cos \lambda_{k} x+\sum_{k=1}^{\infty} u_{2 k}(t) \sin \lambda_{k} x \quad\left(\lambda_{k}=2 \pi k\right),
$$

where

$$
\begin{gathered}
u_{10}(t)=\int_{0}^{1} u(x, t) d x, \\
u_{1 k}(t)=2 \int_{0}^{1} u(x, t) \cos \lambda_{k} x d x, \quad u_{2 k}(t)=2 \int_{0}^{1} u(x, t) \sin \lambda_{k} x d x \quad(k=1,2, \ldots) .
\end{gathered}
$$

Then, applying the formal scheme of the Fourier method, from (1) and (2) we have

$$
u_{10}^{\prime \prime}(t)=F_{10}(t ; u, a) \quad(0 \leq t \leq T)
$$




$$
\begin{array}{cl}
\left(1+\lambda_{k}^{2}\right) u_{i k}^{\prime \prime}(t)+\lambda_{k}^{2} u_{i k}(t)=F_{i k}(t ; u, a) \quad(0 \leq t \leq T ; \quad i=1,2 ; \quad k=1,2, \ldots), & \\
u_{10}(0)=\varphi_{10}, \quad u_{10}^{\prime}(0)=\psi_{10}, \\
u_{i k}(0)=\varphi_{i k}, \quad u_{i k}^{\prime}(0)=\psi_{i k} \quad(i=1,2 ; \quad k=1,2, \ldots),
\end{array}
$$

where

$$
\begin{gathered}
F_{1 k}(t ; u, a)=a(t) u_{1 k}(t)+f_{1 k}(t) \quad(k=0,1, \ldots), \\
f_{10}(t)=\int_{0}^{1} f(x, t) d x, \quad f_{1 k}(t)=2 \int_{0}^{1} f(x, t) \cos \lambda_{k} x d x \quad(k=1,2, \ldots), \\
\varphi_{10}=\int_{0}^{1} \varphi(x) d x, \quad \psi_{10}=\int_{0}^{1} \psi(x) d x, \\
\varphi_{1 k}=2 \int_{0}^{1} \varphi(x) \cos \lambda_{k} x d x, \quad \psi_{1 k}=2 \int_{0}^{1} \psi(x) \cos \lambda_{k} x d x \quad(k=1,2, \ldots), \\
F_{2 k}(t ; u, a)=a(t) u_{2 k}(t)+f_{2 k}(t), \quad f_{2 k}(t)=2 \int_{0}^{1} f(x, t) \sin \lambda_{k} x d x \quad(k=1,2, \ldots), \\
\varphi_{2 k}=2 \int_{0}^{1} \varphi(x) \sin \lambda_{k} x d x, \quad \psi_{2 k}=2 \int_{0}^{1} \psi(x) \sin \lambda_{k} x d x \quad(k=1,2, \ldots) .
\end{gathered}
$$

Solving problem (19)- (22), we find

$$
\begin{gathered}
u_{10}(t)=\varphi_{10}+t \psi_{10}+\int_{0}^{t}(t-\tau) F_{10}(\tau ; u, a) d \tau \quad(0 \leq t \leq T), \\
u_{i k}(t)=\varphi_{i k} \cos \beta_{k} t+\psi_{i k} \frac{1}{\beta_{k}} \sin \beta_{k} t+ \\
+\frac{1}{\beta_{k}\left(1+\lambda_{k}^{2}\right)} \int_{0}^{t} F_{i k}(\tau ; u, a) \sin \beta_{k}(t-\tau) d \tau(i=1,2 ; k=1,2, \ldots ; 0 \leq t \leq T),
\end{gathered}
$$

where

$$
\beta_{k}=\frac{\lambda_{k}}{\sqrt{1+\lambda_{k}^{2}}}(i=1,2 ; k=1,2, \ldots) .
$$

After substituting the expressions $u_{1 k}(t) \quad(k=0,1, \ldots)$ and $u_{2 k}(t) \quad(k=1,2, \ldots)$ into (18), for the component $u(x, t)$ of the solution $\{u(x, t), a(t)\}$ of problem (1) - (3), (6), (7) we get

$$
\begin{aligned}
& u(x, t)=\varphi_{10}+t \psi_{10}+\int_{0}^{t}(t-\tau) F_{10}(\tau ; u, a) d \tau+ \\
&+\sum_{k=1}^{\infty}\left\{\varphi_{1 k} \cos \beta_{k} t+\psi_{1 k} \frac{1}{\beta_{k}} \sin \beta_{k} t+\right. \\
&\left.+\frac{1}{\beta_{k}\left(1+\lambda_{k}^{2}\right)} \int_{0}^{t} F_{1 k}(\tau ; u, a) \sin \beta_{k}(t-\tau) d \tau\right\} \cos \lambda_{k} x+ \\
&+\sum_{k=1}^{\infty}\left\{\varphi_{2 k} \cos \beta_{k} t+\psi_{21 k} \frac{1}{\beta_{k}} \sin \beta_{k} t+\right.
\end{aligned}
$$




$$
\left.+\frac{1}{\beta_{k}\left(1+\lambda_{k}^{2}\right)} \int_{0}^{t} F_{2 k}(\tau ; u, a) \sin \beta_{k}(t-\tau) d \tau\right\} \sin \lambda_{k} x .
$$

Now, from (7) and (18) we have

$$
\begin{gathered}
a(t)=[h(t)]^{-1}\left\{h^{\prime \prime}(t)-f\left(x_{0}, t\right)+\right. \\
+\sum_{k=1}^{\infty}\left[\lambda_{k}^{2} u_{1 k}^{\prime \prime}(t)+\lambda_{k}^{2} u_{1 k}(t)\right] \cos \lambda_{k} x_{0}+ \\
\left.+\sum_{k=1}^{\infty}\left[\lambda_{k}^{2} u_{2 k}^{\prime \prime}(t)+\lambda_{k}^{2} u_{2 k}(t)\right] \sin \lambda_{k} x_{0}\right\} .
\end{gathered}
$$

By (20) and (24) we have

$$
\begin{gathered}
\lambda_{k}^{2} u_{i k}^{\prime \prime}(t)+\lambda_{k}^{2} u_{i k}(t)=F_{i k}(t ; u, a)-u_{i k}^{\prime \prime}(t)= \\
=\beta_{k}^{2} F_{i k}(t ; u, a)+\beta_{k}^{2} \varphi_{i k} \cos \beta_{k} t+\beta_{k} \psi_{i k} \sin \beta_{k} t+ \\
+\frac{\beta_{k}}{1+\lambda_{k}^{2}} \int_{0}^{t} F_{i k}(\tau ; u, a) \sin \beta_{k}(t-\tau) d \tau(i=1,2 ; k=1,2, \ldots) .
\end{gathered}
$$

To obtain the equation for the second component $a(t)$ of the solution $\{u(x, t), a(t)\}$ to the problem (1) - (3), (6), (7), substitute expression (28) into (27) and have

$$
\begin{array}{r}
a(t)=[h(t)]^{-1}\left\{h^{\prime \prime}(t)-f\left(x_{0}, t\right)+\sum_{k=1}^{\infty}\left\{\beta_{k}^{2} F_{1 k}(t ; u, a)+\beta_{k}^{2} \varphi_{1 k} \cos \beta_{k} t+\right.\right. \\
\left.+\beta_{k} \psi_{1 k} \sin \beta_{k} t+\frac{\beta_{k}}{1+\lambda_{k}^{2}} \int_{0}^{t} F_{1 k}(\tau ; u, a) \sin \beta_{k}(t-\tau) d \tau\right\} \cos \lambda_{k} x_{0}+ \\
+\sum_{k=1}^{\infty}\left\{\beta_{k}^{2} F_{2 k}(t ; u, a)+\beta_{k}^{2} \varphi_{2 k} \cos \beta_{k} t+\right. \\
\left.\left.+\beta_{k} \psi_{2 k} \sin \beta_{k} t+\frac{\beta_{k}}{1+\lambda_{k}^{2}} \int_{0}^{t} F_{2 k}(\tau ; u, a) \sin \beta_{k}(t-\tau) d \tau\right\} \sin \lambda_{k} x_{0}\right\}
\end{array}
$$

Thus, the problem (1) - (3), (6), (7) is reduced to solving the system (26), (29) with respect to the unknown functions $u(x, t)$ and $\mathrm{a}(\mathrm{t})$.

Similarly to (Mehraliyev, 2012) it is possible to prove the following lemma.

Lemma 2 If $\{u(x, t), a(t)\}-i$ any solution of problem (1) - (3), (6), (7), then the functions

$$
\begin{gathered}
u_{10}(t)=\int_{0}^{1} u(x, t) d x, \\
u_{1 k}(t)=2 \int_{0}^{1} u(x, t) \cos \lambda_{k} x d x, \quad u_{2 k}(t)=2 \int_{0}^{1} u(x, t) \sin \lambda_{k} x d x \quad(k=1,2, \ldots) .
\end{gathered}
$$

satisfy system (23), (24) in $[0, T]$.

Remark 1 It follows from lemma 2 that to prove the uniqueness of the solution to the problem (1) - (3), (6), (7), it suffices to prove the uniqueness of the solution to the system (26), (29). 
In order to investigate problem (1) - (3), (6), (7), consider the following spaces:

1. Denote by $B_{2, T}^{3}$ (Mehraliyev, 2011) the set of all functions $u(x, t)$ of the form

$$
u(x, t)=\sum_{k=0}^{\infty} u_{1 k}(t) \cos \lambda_{k} x+\sum_{k=1}^{\infty} u_{2 k}(t) \sin \lambda_{k} x \quad\left(\lambda_{k}=2 \pi k\right),
$$

defined on $D_{T}$ such that the functions $u_{1 k}(t)(k=0,1, \ldots), u_{2 k}(t)(k=1,2, \ldots)$ are continuous on $[0, T]$ and

$$
\begin{aligned}
J_{T}(u) \equiv & \left\|u_{10}(t)\right\|_{C[0, T]}+\left(\sum_{k=1}^{\infty}\left(\lambda_{k}^{3}\left\|u_{1 k}(t)\right\|_{C[0, T]}\right)^{2}\right)^{\frac{1}{2}}+ \\
& +\left(\sum_{k=1}^{\infty}\left(\lambda_{k}^{3}\left\|u_{2 k}(t)\right\|_{C[0, T]}\right)^{2}\right)^{\frac{1}{2}}<+\infty
\end{aligned}
$$

The norm on this set is given by

$$
\|u(x, t)\|_{B_{2, T}^{3}}=J_{T}(u)
$$

2. Denote by $E_{T}^{3}$ the space $B_{2, T}^{3} \times C[0, T]$ of the vector-functions $z(x, t)=\{u(x, t), a(t)\}$ with the norm

$$
\|z\|_{E_{T}^{3}}=\|u(x, t)\|_{B_{2, T}^{3}}+\|a(t)\|_{C[0, T]} .
$$

It is known that $B_{2, T}^{3}$ and $E_{T}^{3}$ are Banach spaces.

Now, in the space $E_{T}^{3}$ consider the operator

$$
\Phi(u, a)=\left\{\Phi_{1}(u, a), \Phi_{2}(u, a)\right\},
$$

where

$$
\begin{gathered}
\Phi_{1}(u, a)=\tilde{u}(x, t) \equiv \sum_{k=0}^{\infty} \tilde{u}_{1 k}(t) \cos \lambda_{k} x+\sum_{k=1}^{\infty} \tilde{u}_{2 k}(t) \sin \lambda_{k} x, \\
\Phi_{2}(u, a)=\tilde{a}(t),
\end{gathered}
$$

$\tilde{u}_{10}(t), \tilde{u}_{i k}(t),(i=1,2 ; k=1,2, \ldots)$ and $\tilde{a}(t)$ equal to the right hand sides of (23),(24) and (29), respectively.

It is easy to see that

$$
\frac{1}{\sqrt{2}}<\beta_{k}<1
$$

Taking into account these relations, by means of simple transformations we find

$$
\begin{gathered}
\left\|\tilde{u}_{10}(t)\right\|_{C[0, T]} \leq\left|\varphi_{10}\right|+T\left|\psi_{10}\right|+ \\
+T \sqrt{T}\left(\int_{0}^{T}\left|f_{10}(\tau)\right|^{2} d \tau\right)^{\frac{1}{2}}+T^{2}\|a(t)\|_{C[0, T]}\left\|u_{10}(t)\right\|_{C[0, T]}, \\
\left(\sum_{k=1}^{\infty}\left(\lambda_{k}^{3}\left\|\tilde{u}_{i k}(t)\right\|_{C[0, T]}\right)^{2}\right)^{\frac{1}{2}} \leq 2\left(\sum_{k=1}^{\infty}\left(\lambda_{k}^{3}\left|\varphi_{i k}\right|\right)^{2}\right)^{\frac{1}{2}}+2 \sqrt{2}\left(\sum_{k=1}^{\infty}\left(\lambda_{k}^{3}\left|\psi_{i k}\right|\right)^{2}\right)^{\frac{1}{2}}+ \\
+2 \sqrt{2 T}\left(\int_{0}^{T} \sum_{k=1}^{\infty}\left(\lambda_{k}\left|f_{i k}(\tau)\right|\right)^{2} d \tau\right)^{\frac{1}{2}}+2 \sqrt{2} T\|a(t)\|_{C[0, T]}\left(\sum_{k=1}^{\infty}\left(\lambda_{k}^{3}\left\|u_{i k}(t)\right\|_{C[0, T]}\right)^{2}\right)^{\frac{1}{2}},
\end{gathered}
$$




$$
\begin{gathered}
\|\tilde{a}(t)\|_{C[0, T]} \leq\left\|[\mathrm{h}(\mathrm{t})]^{-1}\right\|_{C[0, T]}\left\{\left\|h^{\prime \prime}(t)-f\left(x_{0}, t\right)\right\|_{C[0, T]}+\right. \\
+\frac{\sqrt{6}}{12} \sum_{i=1}^{2}\left(\sum_{k=1}^{\infty}\left(\lambda_{k}^{3}\left|\varphi_{i k}\right|\right)^{2}\right)^{\frac{1}{2}}+\frac{\sqrt{6}}{12} \sum_{i=1}^{2}\left(\sum_{k=1}^{\infty}\left(\lambda_{k}^{3}\left|\psi_{i k}\right|\right)^{2}\right)^{\frac{1}{2}}+ \\
+\frac{\sqrt{6 T}}{12} \sum_{i=1}^{2}\left(\int_{0}^{T} \sum_{k=1}^{\infty}\left(\lambda_{k}\left|f_{i k}(\tau)\right|\right)^{2} d \tau\right)^{\frac{1}{2}}+\frac{\sqrt{6}}{12} \sum_{i=1}^{2}\left(\sum_{k=1}^{\infty}\left(\lambda_{k}\left\|f_{i k}(t)\right\|_{C[0, T]}\right)^{2}\right)^{\frac{1}{2}}+ \\
\left.+\frac{\sqrt{6}}{12}(1+T)\|a(t)\|_{C[0, T]} \sum_{i=1}^{2}\left(\sum_{k=1}^{\infty}\left(\lambda_{k}^{3}\left\|u_{i k}(t)\right\|_{C[0, T]}\right)^{2}\right)^{\frac{1}{2}}\right\}
\end{gathered}
$$

Suppose that the data of problem (1) - (3), (6), (7) satisfy the following conditions

$1 . \varphi(x) \in C^{2}[0,1], \varphi^{\prime \prime \prime}(x) \in L_{2}(0,1), \varphi(0)=\varphi(1), \varphi^{\prime}(0)=\varphi^{\prime}(1), \varphi^{\prime \prime}(0)=\varphi^{\prime \prime}(1)$.

$2 . \psi(x) \in C^{2}[0,1], \psi^{\prime \prime \prime}(x) \in L_{2}(0,1), \psi(0)=\psi(1), \psi^{\prime}(0)=\psi^{\prime}(1), \psi^{\prime \prime}(0)=\psi^{\prime \prime}(1)$.

3. $f(x, t) \in C\left(D_{T}\right), f_{x}(x, t) \in L_{2}\left(D_{T}\right), f(0, t)=f(1, t) \quad(0 \leq t \leq T)$.

4. $h(t) \in C^{2}[0, T], h(t) \neq 0 \quad(0 \leq t \leq T)$.

Then, from (30)-(32), we get

$$
\begin{aligned}
& \|\tilde{u}(x, t)\|_{B_{2, T}^{3}} \leq A_{1}(T)+B_{1}(T)\|a(t)\|_{C[0, T]}\|u(x, t)\|_{B_{2, T}^{3}}, \\
& \|\tilde{a}(t)\|_{C[0, T]} \leq A_{2}(T)+B_{2}(T)\|a(t)\|_{C[0, T]}\|u(x, t)\|_{B_{2, T}^{3}},
\end{aligned}
$$

where

$$
\begin{gathered}
A_{1}(T)=\|\varphi(x)\|_{L_{2}(0,1)}+T\|\psi(x)\|_{L_{2}(0,1)}+T \sqrt{T}\|f(x, t)\|_{L_{2}\left(D_{T}\right)}+ \\
+4\left\|\varphi^{\prime \prime \prime}(x)\right\|_{L_{2}(0,1)}+4 \sqrt{2}\left\|\psi^{\prime \prime \prime}(x)\right\|_{L_{2}(0,1)}+4 \sqrt{2 T}\left\|f_{x}(x, t)\right\|_{L_{2}\left(D_{T}\right)}, \\
B_{1}(T)=T^{2}+2 \sqrt{2} T, \\
A_{2}(T)=\left\|[\mathrm{h}(\mathrm{t})]^{-1}\right\|_{C[0, T]}\left\{\| h^{\prime \prime}(t)-f\left(x_{0}, t\right)\right)\left\|_{C[0, T]}+\frac{\sqrt{6}}{6}\right\| \varphi^{\prime \prime \prime}(x) \|_{L_{2}(0,1)}+ \\
\left.+\frac{\sqrt{6}}{6}\left\|\psi^{\prime \prime \prime}(x)\right\|_{L_{2}(0,1)}+\frac{\sqrt{6 T}}{6}\left\|f_{x}(x, t)\right\|_{L_{2}\left(D_{T}\right)}+\frac{\sqrt{6}}{6}\|\| f_{x}(x, t)\left\|_{C[0, T]}\right\|_{L_{2}(0,1)}\right\}, \\
B_{2}(T)=\left\|[\mathrm{h}(\mathrm{t})]^{-1}\right\|_{C[0, T]} \frac{\sqrt{6}}{12}(1+T) .
\end{gathered}
$$

It follows from the inequalities (33), (34) that

$$
\|\tilde{u}(x, t)\|_{B_{2, T}^{3}}+\|\tilde{a}(t)\|_{C[0, T]} \leq A(T)+B(T)\|a(t)\|_{C[0, T]}\|u(x, t)\|_{B_{2, T}^{3}},
$$

where

$$
A(T)=A_{1}(T)+A_{2}(T), B(T)=B_{1}(T)+B_{2}(T) .
$$

Now we can prove the following theorem.

Theorem 1. Let the conditions 1-4 be fulfilled and

$$
(A(T)+2)^{2} B(T)<1 .
$$


Then the problem (1) - (3), (6), (7) has a unique solution in the ball $K=K_{R}\left(\|z\|_{E_{T}^{3}} \leq R=A(T)+2\right)$ of the space $E_{T}^{3}$.

Remark 2. Inequality (36) is satisfied for sufficiently small values at $T+\left\|h^{-1}(t)\right\|_{C[0, T]}$.

Proof. In the space $E_{T}^{3}$ consider the equation

$$
z=\Phi z
$$

where $z=\{u, a\}$ and the components $\Phi_{i}(u, a)(i=1,2)$ of the operator $\Phi(u, a)$ are given by the right hand sides of the equations (26), (29). Consider the operator $\Phi(u, a)$ in the ball $K=K_{R}$ from $E_{T}^{3}$. Similar to (35), we see that for any $z, z_{1}, z_{2} \in K_{R}$ the following estimates hold:

$$
\begin{gathered}
\|\Phi z\|_{E_{T}^{3}} \leq A(T)+B(T)\|a(t)\|_{C[0, T]}\|u(x, t)\|_{B_{2, T}^{3}}, \\
\left\|\Phi z_{1}-\Phi z_{2}\right\|_{E_{T}^{3}} \leq B(T) R\left(\left\|a_{1}(t)-a_{2}(t)\right\|_{C[0, T]}+\left\|u_{1}(x, t)-u_{2}(x, t)\right\|_{B_{2, T}^{3}}\right) .
\end{gathered}
$$

Then, it follows from (36) together with the estimates (38) and (39) that the operator $\Phi$ acts in the ball $K=K_{R}$ and is contractive. Therefore, in the ball $K=K_{R}$ the operator $\Phi$ has a unique fixed point $\{u, a\}$, that is a unique solution of equation (37) in the ball $K=K_{R}$, i.e. it is a unique solution of system (26), (29) in the ball $K=K_{R}$.

The function $u(x, t)$, as an element of the space $B_{2, T}^{3}$ is continuous and has continuous derivatives $u_{x}(x, t)$ and $u_{x x}(x, t)$ in $D_{T}$.

Further, from (20) it follows that $u_{i k}^{\prime \prime}(t)(i=1,2 ; k=1,2, \ldots)$ is continuous in $[0, T]$ and consequently we have:

$$
\begin{gathered}
\left(\sum_{k=1}^{\infty}\left(\lambda_{k}^{3}\left\|u_{i k}^{\prime \prime}(t)\right\|_{C[0, T]}\right)^{2}\right)^{\frac{1}{2}} \leq \sqrt{2}\left(\sum_{k=1}^{\infty}\left(\lambda_{k}^{3}\left\|u_{i k}(t)\right\|_{C[0, T]}\right)^{2}\right)^{\frac{1}{2}}+ \\
+\sqrt{2}\|\| f(x, t)+a(t) u(x, t)\left\|_{C[0, T]}\right\|_{L_{2}(0,1)}(i=1,2) .
\end{gathered}
$$

From the last relation it is obvious that $u_{t t}(x, t), u_{t t x}(x, t), u_{t t x x}(x, t)$ is continuous in $D_{T}$.

It is easy to verify that the equation (1) and conditions (2), (3), (6), (7) are satisfied in the ordinary sense.

Consequently, $\{u(x, t), a(t)\}$ is a solution of problem (1)-(3), (6), (7), and by lemma 2 it is unique in the ball $K=K_{R}$. The theorem is proved.

By lemma 1 the unique solvability of the initial problem (1)-(5) follows from the theorem.

Theorem 2. Let all the conditions of theorem 1 be fulfilled and

$$
\begin{gathered}
\int_{0}^{1} f(x, t) d x=0 \quad(0 \leq t \leq T), \\
\int_{0}^{1} \varphi(x) d x=0, \quad \int_{0}^{1} \psi(x) d x=0, \quad \varphi\left(x_{0}\right)=h(0), \quad \psi\left(x_{0}\right)=h^{\prime}(0) .
\end{gathered}
$$

Then the problem (1)- (5) has a unique classical solution in the ball $K=K_{R}\left(\|z\|_{E_{T}^{3}} \leq R=A(T)+2\right)$ of the space $E_{T}^{3}$.

\section{References}

Budak, B .M., Samarskii, A. A., \& Tikhonov, A. N. (1972). Collector of tasks in mathematical physics, M.: Nauka (in Russian)

Denisov, A. M. (1994). Introduction to theory of inverse problems. MSU.

Gabov, S. A., \& Orazov, B. B. (1986). The equation $\frac{\partial^{2}}{\partial t^{2}}\left[u_{x x}-u\right]+u_{x x}=0$ and several problems associated with it . Dokl. Computational Mathematics and Mathematical Physics, Vol.26, No 1, pp. 92-102 (in Russian) 
Ivanov, V. K., Vasin V. V., \& Tanina, V. P. (1978). Theory of linear ill-posed problems and its applications. M.: Nauka (in Russian)

Lavrent'ev, M. M. (1964). On an inverse problem for a wave equation. Dokl. AN SSSR, Vol.157, No 3 , pp. 520-521 (in Russian)

Lavrent'ev, M. M., Romanov, V. G., \& Shishatsky, S. T. (1980). Ill-posed problems of mathematical physics and analysis. M.: Nauka (in Russian)

Mehraliyev, Ya. T. (2011). Inverse boundary problem for a partial differential equation of fourth order with integral condition. Dokl. South Ural State University Herald. Series: "Mathematics, Mechanics, Physics, Vol.32(249), No 5 , pp. 51-56 (in Russian)

Mehraliyev, Ya. T. (2011). On one inverse boundary value problem for hyperbolic equations of second order with integral condition of the first kind . Dokl. The Bryansk State University Herald No 4, pp. 22-28 (in Russian)

Mehraliyev, Ya. T. (2011). On solvability of an inverse boundary value problem for a second order elliptic equation. Dokl. Vestnic Tverskogo Gosudarstvennogo Universiteta. Ser. prikladnaya matematika No 23 , pp. 25-38 (in Russian)

Mehraliyev, Ya. T. (2012). Inverse boundary value problem for a second order elliptic equation with additional integral condition. Dokl. Vestnik of Udmurtskogo Universiteta. Mathematika. Mekhanika. Komputerniye Nauki, Issue 1, pp. 32-40 (in Russian)

Tikhonov, A. N. (1943). On stability of inverse problems. Dokl. AN SSSR, 39(5), 195-198 (in Russian)

\section{Copyrights}

Copyright for this article is retained by the author(s), with first publication rights granted to the journal.

This is an open-access article distributed under the terms and conditions of the Creative Commons Attribution license (http://creativecommons.org/licenses/by/3.0/). 